\title{
Assessing and understanding hospitality: The Brief Hospitality Scale
}

\author{
Robert Biswas-Diener · Kostadin Kushlev · Rong Su · Fallon Goodman • \\ Todd B. Kashdan · Ed Diener
}

\begin{abstract}
Although hospitality is a valued social and cultural phenomenon, it has been largely overlooked in the psychology research literature. Our studies are designed to advance the understanding of hospitality by creating a brief measure of it that can be used across cultures. In Study 1, we employed a large sample of Americans to create and begin validation of a measure of hospitality: the Brief Hospitality Scale, or BHS. In all nations and both studies, the scale had a single strong factor and high internal consistency. In Study 2, we administered the measure to respondents from 11 nations and found that people in some countries (e.g., Iran) are significantly more hospitable than people in others (e.g., Singapore). The strongest personality correlates of hospitality were those associated with social characteristics such as extraversion, agreeableness, and feelings of group belonging. The very strongest association with hospitality was the ability to see the perspective of others. Thus, hospitality represents more than simple sociability, and seems to rest on feelings of togetherness with others, concern for their wellbeing, and positive feelings toward them. We found in both studies that hospitality is associated with higher levels of wellbeing, for example, optimism, psychosocial flourishing, and positive affect.
\end{abstract}

Keywords: hospitality, personality, subjective wellbeing, perspective taking, positive psychology measurement

\section{Introduction}

Hospitality is ubiquitous in daily life. Whenever we have friends over for dinner, host an old classmate for a few days, or throw a barbeque for neighbors, we engage in hospitable behavior. Though such large and small social occasions likely play a role in the maintenance of social relationships and the development of community, hospitality has been largely overlooked by scholars of psychology in general, and wellbeing scholars in particular. In this initial exploration of hospitality, we create a measure of hospitality, examine its correlates to gain a better understanding of it, and assess its variation across cultures.

While social behavior is an essential factor in subjective wellbeing (Diener \& Seligman, 2002; Epley \& Schroeder, 2014; Kahneman, Krueger, Schkade, Schwarz, \& Stone, 2004; Reis, Sheldon, Gable, Roscoe, \& Ryan, 2000; Sandstrom \& Dunn, 2014), hospitality does not appear in the indexes of three widely used textbooks in positive psychology (Baumgardner \& Crothers, 
2009; Peterson, 2006; Snyder, Lopez, \& Pedrotti, 2011), or among the 55 chapters collected in the second edition of the Handbook of positive psychology (Snyder \& Lopez, 2002). Similarly, hospitality is not listed among the 24-character strengths of the Values in Action survey (Peterson \& Seligman, 2004), often regarded as central elements of psychological wellbeing. Beyond positive psychology, hospitality does not even appear in the indexes of popular introductory psychology textbooks. As Rozin (2006) has pointed out, however, behavioral scientists need to focus more on important behaviors that characterize everyday life. This certainly applies to the topic of hospitality, which exists across cultures, economic status, and throughout history.

Like happiness and empathy, hospitality may be a concept with nuanced and diverse definitions. The Oxford English dictionary defines hospitality as "friendly and generous reception and entertainment of guests or strangers" and also as "cordiality, warmth, congeniality, sociability and generosity." These two definitions suggest the complexity of the concept. On the one hand, hospitality is viewed in behavioral terms. It is a transaction between a host and a guest in which the former shares personal resources with the latter. For example, a host provides dinner or entertainment for a guest. On the other hand, as suggested by the second definition, hospitality is also viewed as an attitude. In this case, it is understood to be a quality of personality: an attitude of welcoming.

There are two hallmark features of hospitality. The first is the physical location of the pro-social interaction. In the case of hospitality, sharing-broadly defined-happens within the context of the host's home ("home" here could mean office, house, or other locations that constitute the host's-but not the guest's - territory). That is, the host welcomes the guest into the host's territory, where the sharing occurs. As such, hospitality is a face-to-face (whether virtual or physical) phenomenon that does not allow for anonymity in the relationship. The face-to-face aspect of hospitality is non-trivial because it relates to the second hallmark feature: the social and emotional tenor of the transaction, namely, hospitality is concerned with fostering a relaxed and positive tone. Historically, strangers, travelers, and even enemies enjoyed occasional hospitality governed by formal rules. For example, Hávamál, "The sayings of the Vikings," provides a historical example of the specifics of hospitality with an emphasis on positivity:

The newcomer needs fire / his knees are numb. / A man who has made his way over mountains needs food and fresh linen.... A guest needs giving water / fine towels and friendliness. / A cheerful word / a chance to speak / kindness and concern. (Jónasson, 1992, pp. 20-21).

It should be noted that hospitality - in the lay understanding of the concept-is also distinct from related concepts, such as generosity, even though both concepts fall under the broad umbrella of "pro-social behavior." Generosity is a relatively liberal attitude toward the giving of one's own resources, including money and possessions. Generosity is distinct from hospitality in that it can include anonymous transactions (for example, donating money to aid organizations). In addition, generosity has no element of welcome. Purchasing a theater ticket for a friend who forgot her wallet, for instance, is generous but not-by definition - hospitable. Further, it is possible that hospitality does not always include the giving of one's resources on a permanent basis. For example, a person who invites her neighbors over after their house is flooded provides temporary shelter. These commonsense understandings of hospitality suggest 
that it is a distinct, albeit multi-faceted, aspect of pro-social behavior. Research on this topic can help us further disentangle and understand hospitality.

When researchers have examined the concept of hospitality, it has largely been within the scope of the hospitality industry. This industry includes businesses such as hotels and restaurants that provide food, shelter, and-to some degree-entertainment of guests. It should be noted, however, that the hospitality industry is fundamentally inadequate to define or describe hospitality as it occurs in everyday life. This is because the hospitality industry treats hospitality as a financial transaction. That is, the hospitality industry promotes a welcoming attitude and a focus on guest satisfaction - both psychological concepts - but it is motivated primarily by guest loyalty, and, by extension, profit (Walker, 2009). As such, the welcoming attitude and helpful behaviors of people in the hospitality industry may not reflect noncommercial hospitality. That said, research from the hospitality industry might provide some insights into this phenomenon. This can be seen, for example, in the concept of "service orientation" (Hogan, Hogan, \& Busch, 1984; Dienhart, Gregoire, Downey, \& Knight, 1992). Service orientation is broadly defined as a disposition toward helpfulness and cooperation, but specifically in the context of customer service, such as restaurant interactions. Customer service bears a passing similarity to the concerns of hosts interested in welcoming guests. Pezzotti (2011) argues, however, that there are subtle but important distinctions between hospitality and service. He associates the words "warm," "friendly," "listening," "respect," and "security" with hospitality and "scripted," "standards," "products," and "anticipation" with service. He points out the ways in which service is artificial and oriented toward pleasing customers, while hospitality is organic and oriented toward social connection. Thus, while existing research has yielded important applied insights for the hospitality industry, a large gap remains in our basic understanding of the psychological process of individual hospitality as it occurs in everyday life. Although psychologists have studied cooperation, altruism, generosity, and related concepts, there is neither a robust literature on hospitality nor established theories or models.

\section{The current research}

In this article, we have three primary goals: (1) create a valid brief measure of hospitality, (2) explore its associations with personality and wellbeing, and (3) use it as an instrument to explore differences between people from nations across the globe. Overall, we hope that this initial exploration of the concept of hospitality will bring the topic of hospitality to the attention of behavioral researchers, who will build on our work by further refining understanding the role of hospitality in social life.

We began with a pilot study with open-ended responses, in which we gained initial insights into the content and facets of hospitality by polling two separate samples: one sample focusing on experiences related to being a host ( $\mathrm{N}=105 ; 74 \%$ female) and a second one focusing on experiences related to being a guest $(\mathrm{N}=65 ; 75 \%$ female). Across the two samples, participants came from 15 diverse countries (e.g., the United States, Turkey, Portugal, India), allowing us to identify possible societal and cultural aspects of the hospitality experience. Based on these survey responses, we created an initial pool of 68 hospitality items, which we later reduced to 23 items. From the 23 items we selected four that formed a single factor, and yet tapped into somewhat different aspects (e.g., enjoyment, self-perception) of hospitality - the Brief Hospitality Scale (BHS; see Study 1 below).

We explored the BHS by its associations with personality, culture, and wellbeing in a large sample of Americans (Study 1) and in an international sample of individuals across 11 
countries (Study 2). We predicted strong positive association between hospitality and socially relevant personality traits such as extroversion and agreeableness, helping to establish the convergent validity of the scale. As an additional factor that should be particularly highly associated with hospitality, we assessed perspective taking (Hodges \& Biswas-Diener, 2007), which has been linked to empathic concern and altruistic behavior (Batson, Early, \& Salvarani, 1997). In contrast to the social personality traits, conscientiousness, openness, and neuroticism should display weaker associations with hospitality -indicating the discriminant validity of the scale. Further, as social behavior plays a key role in promoting subjective wellbeing (e.g., Diener \& Seligman, 2002; Reis et al., 2000), hospitality should predict higher wellbeing.

Finally, we further examined the BHS by assessing individuals from various nations in Study 2's international sample. Though not using a validated scale of hospitality, a survey of 18,135 expatriates asked to report how welcome they felt in various countries suggests that hospitality varies substantially across nations (InterNations, 2018); a valid measure of hospitality should, therefore, be able to reveal such cultural differences.

\section{Study 1: Measure creation}

The primary purpose of this study was to create a hospitality scale that includes affective and attitudinal items to measure hospitality. Each item was constructed as a statement that respondents responded to by indicating their amount of relative agreement using a 1 (total disagreement) to 7 (total agreement) Likert scale, with 4 indicating a neutral middle point. It was our aim to create an initial measure of the construct of hospitality while also examining this concept in relation to other psychological variables of interest, including wellbeing and personality.

\subsection{Method}

Participants. Participants ( $N=1,623 ; 50 \%$ female) were recruited online through Qualtrics data collection services, allowing us to collect data from a diverse sample of people living in the United States. The respondents' ages ranged from 18 to 70 years old $(M=48.62, S D=12.89)$. The participants represented a wide range of work backgrounds, including trades, professions, students, retirees, and non-workers.

Measures. We created an initial pool of 68 items that attempted to capture diverse aspects of hospitality, including diverse feelings and beliefs about it. For example, we were curious about the extent to which people enjoy hospitality, see it as a moral undertaking, and see it as an obligation. Responses to each item were answered on a 7-point scale ranging from 1 (strongly disagree) to 7 (strongly agree). Our original intent was to create a general hospitality scale with distinct subscales, but factor analyses of the items revealed no clear-cut simple and understandable structure beyond a single strong first factor. The four items we ultimately chose for the Brief Hospitality Scale were selected based on the following criteria: a) unidimensionality, b) strong alpha coefficients, c) the inclusion of diverse psychological aspects of hospitality (enjoyment, self-esteem, naturalness, and identity), and d) experiences that would be general across nations.

In addition to the hospitality items, participants responded to the Satisfaction with Life Scale (SWLS; Diener, Emmons, Larsen, \& Griffin, 1985; Pavot \& Diener, 1993). Respondents also completed the Scale of Positive and Negative Experience - a validated measure of positive and negative affect (SPANE; Diener, et al., 2009). Participants also responded to the Mini-IPIP-a short measure of Big 5 personality traits that shows both adequate psychometric properties and 
convergence with other widely used measures of personality (Donnellan, Oswald, Baird, \& Lucas, 2006). We also included four items related to perspective taking. Perspective taking is the cognitive aspect of empathy, and is related to a person's ability to see the world from another person's perspective (Hodges \& Biswas-Diener, 2007). Finally, we assessed the income, education, age, and gender of our participants.

\subsection{Results}

We factor analyzed the full set of items and selected items that loaded highly on the first factor but assessed somewhat different content. After identifying four items as promising, we subjected them to a principal components analysis, as well as a scale reliability analysis (Cronbach's alpha). There was a strong single factor (strictly speaking, a single component, as we employed a principal components analysis), with no second factors having an eigenvalue even approaching 1.0. The individual item loadings are shown in Table 1. A maximum likelihood factor analysis produced virtually identical conclusions, with one very strong factor and no other factor approaching one. The Cronbach's alpha for the scale was .87 , with all interitem correlations exceeding .60.

\section{Table 1. Principal component loadings and Cronbach's Alpha of the BHS}

$\begin{array}{llr}\text { Items } & \text { Study } 1 & \text { Study } 2\end{array}$

\begin{tabular}{lll}
\hline I enjoy hosting & .87 & .84 \\
I feel good about myself when hosting & .88 & .87 \\
Being hospitable comes easily to me & .84 & .91 \\
I see myself as hospitable & .83 & .91
\end{tabular}

The scale is scored by summing the four items. The four-item Brief Hospitality Scale (BHS) is shown in Appendix 1. Scale scores can vary from 4 to 28 (the sum of four items that can range from 1 to 7 each). In Table 1, we present the weightings for the four items in both studies, derived from the principal components analysis. It should be noted that when the maximum likelihood factor analysis was employed, virtually the same weights were derived. As can be seen, all four items weighted highly on the single dimension, and were of similar magnitude.

The means and standard deviations for the scale for all respondents in Study 1, as well as for demographic groups, are shown in Table 2. The scale scores were negatively skewed (-.51), with only a few individuals scoring very low, but relatively more individuals scoring high on hospitality. As can be seen, the scale scores averaged about 19.5, meaning a score of about 5 per item, or "slightly agree." As can also be seen, the various demographic groups did not vary dramatically from each other in hospitality. The only significant difference between groups was that women were more hospitable than men, $t(1592)=5.40, p<.001)$. 
Table 2. Descriptive statistics for the four-item Brief Hospitality Scale for the USA

\begin{tabular}{lclc}
\hline & Valid N & Mean & Standard Deviation \\
Entire Sample & 1602 & 19.5 & 5.0 \\
Women & 801 & 20.1 & 5.0 \\
Men & 793 & 18.8 & 4.9 \\
Married & 894 & 19.6 & 5.0 \\
Not Married & 698 & 19.2 & 5.0 \\
Young (18-35) & 314 & 19.5 & 4.8 \\
Middle-aged (36-54) & 630 & 19.1 & 5.0 \\
Senior (55-70) & 587 & 19.7 & 5.1 \\
\hline
\end{tabular}

Correlations with other measures. The correlations between the BHS and demographic, personality traits, and wellbeing are shown in Table 3. Providing evidence of convergent validity, hospitality was highly correlated with social dimensions of personality, such as extraversion and agreeableness, and very highly with perspective taking. Hospitality was also significantly associated with each form of subjective wellbeing.

Hospitality was seen as good and moral in the USA. The mean response to "Hospitable people are good people" was 4.96, and to "It is morally right to be hospitable" was 5.15. Thus, there was agreement that hospitality is desirable, but it was only mild agreement, not rising to a strong imperative.

Table 3. Correlations of hospitality with personality, wellbeing, and demographic variables

\begin{tabular}{rrr} 
& Study 1 & Study 2 \\
\hline
\end{tabular}

Five Factor Personality Traits

Extroversion $\quad .45$

.49

Agreeableness

.40

Conscientiousness

.50

.19

Neuroticism

Openness

$-.26$

Perspective Taking

.69

Wellbeing

Life Satisfaction

Positive Affect

Negative Affect

Optimism

Flourishing Scale

\section{Social Thriving}

Social Support

Help Community

Trust

Respected

Lonely

$-.26$

Belonging

.43

Note: All correlations $\mathrm{p}<.001$ 
In order to further test the validity of the BHS, we asked about four specific and concrete hospitable behaviors, in order to see if our scale could predict them. In Table 4, we show the correlations between the BHS and these four behavioral items. The table reveals moderate to strong correlations between BHS scores and hospitable behaviors.

Table 4. BHS scores correlated with four hospitable behaviors

\begin{tabular}{lll}
\hline & Study $\mathbf{1}$ & Study $\mathbf{2}$ \\
\hline I regularly play host to my family members & .53 & .53 \\
\hline I have hosted a stranger at a dinner party & .45 & .39 \\
\hline I have recently had a guest spend the night in my home & .40 & .39 \\
\hline I host a dinner or a party where I live at least once a month & .35 & .51 \\
\hline
\end{tabular}

Note: All correlations are significant at $p<.001$

\subsection{Discussion}

In Study 1, we created a four-item scale of hospitality that forms a single factor with very high internal reliability. The scale correlates substantially with perspective taking, as well as with extroversion and agreeableness. Thus, the scale correlates with individual differences in personality related to sociability and being able to get along well with others. Furthermore, high hospitality was correlated with higher subjective wellbeing, including life satisfaction. The association with positive affect was notably higher than with negative affect.

\section{Study 2: Cross-cultural hospitality}

Though Study 1 relied on a broad sample of Americans, the conclusions may not generalize beyond the United States. In Study 2, we address this concern by using samples from 11 different nations and five continents. These samples allowed us to explore cross-national differences.

\subsection{Methods}

Participants. Respondents from 11 nations were recruited to participate through Qualtrics data collection services, $\mathrm{N}=2,145$ (52\% female). Across nations, the average age of the respondents was $M=37.66$ years old, $S D=1.80$. We selected these nations because their societies represent a wide range of historical, cultural, and geopolitical regions and influences.

Measures. Participants responded to the BHS questions related to personality and wellbeing, and questions about their social quality of life. The 18 social items covered a wide range of social wellbeing dimensions including social support, trusting others, and feeling respected. The respondents also answered items assessing subjective wellbeing (optimism, life satisfaction, positive affect, and negative affect). Finally, participants responded to the Flourishing Scale (FS; Diener et al., 2009). The FS is a short composite scale measuring an array of wellbeing variables including self-esteem, purpose, and mastery. It has been shown to have high reliability and high convergence with other wellbeing measures (Diener et al., 2009). Means, standard deviations, and Cronbach's alphas are shown for each of the 11 nations in Table 5. 
Table 5. Descriptive statistics for 11 nations, and scale reliability

\begin{tabular}{lllll}
\hline Nation & N & Mean & SD & $\begin{array}{l}\text { Cronbach's } \\
\text { Alpha }\end{array}$ \\
\hline Argentina & 204 & & & \\
Australia & 194 & 20.8 & 5.3 & .87 \\
China & 205 & 18.4 & 5.7 & .92 \\
Germany & 199 & 20.6 & 5.1 & .94 \\
India & 196 & 20.4 & 5.5 & .89 \\
Iran & 149 & 21.0 & 5.4 & .92 \\
Mexico & 196 & 23.5 & 3.8 & .84 \\
Russia & 198 & 20.8 & 5.4 & .86 \\
Singapore & 196 & 19.4 & 6.0 & .94 \\
Spain & 202 & 16.8 & 5.3 & .91 \\
Turkey & 195 & 19.7 & 4.6 & .81 \\
\hline
\end{tabular}

\subsection{Results}

The factor loadings for the four items for Study 2 are shown in Table 1. There was a single strong factor (component) with an eigenvalue above 1. Overall, the four items demonstrated excellent internal consistency, $\alpha=.95$, as in Study 1 . When maximum likelihood factor analysis was applied to the data instead of principal components, the results were identical - with only one very strong factor in each nation, and no others approaching an eigenvalue of 1 .

The correlations of general hospitality with personality and wellbeing are shown in Table 3. As can be seen, hospitality again was strongly associated with extroversion and agreeableness. For social wellbeing, hospitality was most strongly correlated with belonging and helping one's community. Finally, hospitality was very strongly related to positive forms of wellbeing - life satisfaction, positive affect, optimism, and flourishing, and modestly associated inversely with negative affect.

We next explored patterns of differences between nations in hospitality. Assuming that national and cultural differences in hospitality exist, a measure of hospitality with good validity should be able to assess such differences. A visual inspection of the data indicates that some nations, such as Iran, score substantially higher on hospitality than other nations, such as Singapore. 
Table 6. Percentile norms for the Brief Hospitality Scale for the USA (Study 1) and 11 other nations (Study 2)

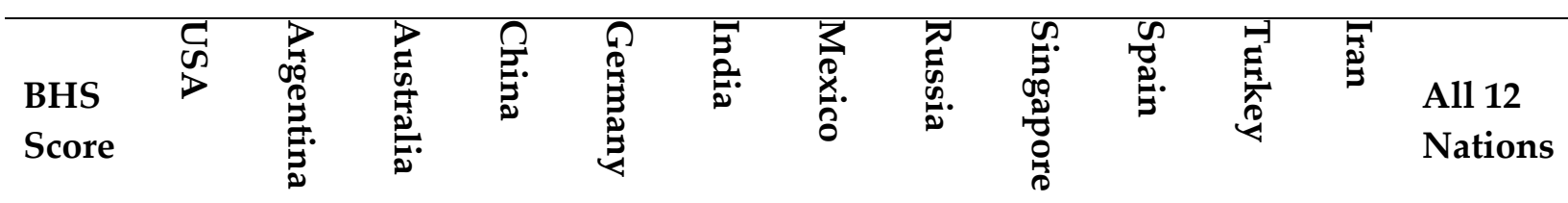

\begin{tabular}{llllllllllllll}
\hline 4 & 1 & 2 & 3 & 1 & 3 & 1 & 2 & 2 & 2 & 1 & 2 & 0 & 1 \\
5 & 1 & 3 & 4 & 1 & 3 & 2 & 2 & 3 & 3 & 2 & 2 & 0 & 2 \\
6 & 2 & 3 & 4 & 2 & 5 & 2 & 3 & 3 & 4 & 2 & 2 & 0 & 2 \\
7 & 2 & 3 & 5 & 3 & 6 & 3 & 4 & 4 & 5 & 3 & 3 & 0 & 3 \\
8 & 3 & 3 & 6 & 3 & 6 & 3 & 4 & 8 & 7 & 3 & 5 & 0 & 4 \\
9 & 4 & 4 & 8 & 4 & 7 & 4 & 4 & 9 & 8 & 3 & 6 & 0 & 5 \\
10 & 5 & 4 & 10 & 4 & 7 & 5 & 6 & 11 & 12 & 4 & 9 & 0 & 6 \\
11 & 6 & 4 & 13 & 5 & 8 & 7 & 6 & 13 & 16 & 4 & 12 & 0 & 7 \\
12 & 8 & 6 & 16 & 7 & 9 & 8 & 7 & 17 & 20 & 5 & 13 & 1 & 9 \\
13 & 10 & 8 & 17 & 8 & 11 & 10 & 9 & 18 & 23 & 9 & 16 & 2 & 11 \\
14 & 13 & 10 & 21 & 12 & 12 & 13 & 11 & 20 & 29 & 11 & 18 & 3 & 14 \\
15 & 18 & 14 & 25 & 15 & 14 & 15 & 15 & 23 & 38 & 18 & 18 & 4 & 18 \\
16 & 30 & 19 & 37 & 20 & 19 & 20 & 21 & 27 & 52 & 26 & 25 & 6 & 27 \\
17 & 36 & 24 & 43 & 25 & 22 & 24 & 25 & 33 & 57 & 30 & 28 & 7 & 32 \\
18 & 42 & 32 & 49 & 30 & 27 & 27 & 29 & 39 & 65 & 36 & 30 & 13 & 38 \\
19 & 47 & 35 & 52 & 36 & 35 & 32 & 34 & 46 & 69 & 44 & 35 & 16 & 43 \\
20 & 55 & 43 & 63 & 48 & 45 & 42 & 41 & 52 & 74 & 55 & 42 & 23 & 52 \\
21 & 63 & 50 & 69 & 53 & 52 & 46 & 50 & 58 & 80 & 61 & 43 & 28 & 58 \\
22 & 69 & 59 & 73 & 58 & 60 & 53 & 54 & 62 & 84 & 68 & 49 & 34 & 64 \\
23 & 75 & 67 & 79 & 64 & 69 & 58 & 64 & 69 & 86 & 77 & 55 & 41 & 71 \\
24 & 86 & 76 & 86 & 76 & 79 & 75 & 76 & 83 & 94 & 91 & 72 & 49 & 82 \\
25 & 90 & 80 & 91 & 82 & 84 & 81 & 82 & 85 & 96 & 94 & 77 & 64 & 87 \\
26 & 93 & 83 & 95 & 90 & 88 & 85 & 84 & 88 & 97 & 95 & 85 & 74 & 90 \\
27 & 95 & 88 & 96 & 95 & 95 & 87 & 91 & 91 & 98 & 97 & 87 & 85 & 93 \\
28 & 100 & 100 & 100 & 100 & 100 & 100 & 100 & 100 & 100 & 100 & 100 & 100 & 100 \\
\hline
\end{tabular}

Note: Scores range from 4 through 28. Nation numbers indicate the percentage of people at or below that BHS score in each nation.

In Table 6, we provide norms for hospitality for each of the 12 nations included in both studies, as well as norms for the entire sample of 12 nations. The differences between mean levels of hospitality are reflected in these norms. For instance, a score of 16 on the BHS would be only the $6^{\text {th }}$ percentile in Iran, but the $52^{\text {nd }}$ percentile in Singapore. Similarly, a score of 20 would be the $23^{\text {rd }}$ percentile in Iran, but the $63^{\text {rd }}$ percentile in Australia. Overall, nations differed significantly $(F(10,2124)=17.77, p<.001)$ in hospitality. In terms of specific comparisons between nations, as analyzed by Bonferroni comparisons, the results can be summarized as: Iran was significantly higher than all other nations $(p<.001)$, and Singapore and Australia were significantly lower $(p<.01)$ than most other nations, but not each other. 
In terms of demographic differences in Study 2, women were significantly $(\mathrm{t}=2.51, \mathrm{p}<$ .05) higher than men (20.1 versus 19.4), as in Study 1. Married individuals scored higher on hospitality compared to other marital status groups (20.6 versus $19.6 ; t=3.99, p<.001)$. In the sample of 11 nations, age differences were more pronounced than in Study 1: Young group $=$ 19.4; middle-aged group $=20.1$; and the senior group $=20.8(\mathrm{~F}(2,1979)=9.17, \mathrm{p}<.001)$. Thus, across the two studies, women, married people, and seniors tended to be more hospitable than their counterparts. Nonetheless, demographic differences did not appear large in absolute terms.

As in Study 1, we analyzed whether people in the 11 nations believe that hospitality is good and moral. In every nation and across the 11 nations hospitality was rated above the neutral point of 4 for both "It is morally right to be hospitable" and "Hospitable people are good people." These numbers were, however, notably higher in nations such as Iran (6.47 and 6.11) than in nations such as Australia (4.94 and 4.73) and Singapore (4.57 and 4.35). Thus, hospitality is universally seen as desirable, but the strength of this varies across nations. It appears that hospitality is lower in nations where it is less valued.

\section{General discussion}

The current study offers an initial exploration into the experience of hospitality. This is a common social phenomenon that has, until now, been conspicuously absent from psychological research attention. Our investigation aimed to better understand a variety of psychological components of hospitality, including identity (a sense of self as hospitable), affect (enjoying hospitality), effort (whether it comes easily), and self-esteem (hospitality makes one feel good about oneself). We created a brief measure of hospitality and administered it in 12 nations.

To create the BHS measure of hospitality, we began with a pilot study into people's experiences of being guests and of being hosts. We used these insights to create an item pool aimed at capturing the various aspects of hospitality. Next, we used a diverse sample of more than 1,000 Americans to create and test a four-item measure that shows robust psychometric properties. The scale was unidimensional and had a strong Cronbach's alpha in all 12 nations, and we later used it to collect additional cross-national data.

We used the BHS to examine common personality predictors, including the Five Factor model and perspective taking. Of the Five Factors, extroversion was the strongest predictor of overall hospitality. It may be that extroverts are particularly suited to the social aspects of hospitality (Grobelna, 2015). As expected, perspective taking was also very strongly associated with overall hospitality. It may be that the ability to "put one's self in another person's shoes" is a psychological skill that makes hospitality easier and more effective. Further investigation of the ways that individual differences affect hospitality is highly recommended to future researchers.

The Brief Hospitality Scale was also used to investigate cross-national differences in hospitality. First, we collected data from diverse samples from geographically distinct countries and found that hospitality differs from society to society. Indeed, while the idea that hospitality is a virtuous action seemed to be a cultural universal, we found mean rates and distributions of hospitality to differ from nation to nation. Middle-Eastern nations (such as Iran and Turkey) 
and Latin countries (such as Mexico and Argentina) reported the highest rates of hospitality. We also found that norms help interpret these scores by revealing how high a score is compared to other individuals in that nation, as well as contrasted against the norms for the other nations. Although we found differences in this preliminary study, we acknowledge that we know too little, at present, to meaningfully draw conclusions about cultural factors that might influence these differences. It is possible, for example, that the low scores found in Singapore reflect unique cultural factors such as a lower value placed on hospitality, the potential impact of reputation concerns on hospitality, the influence of collectivism, or some other factor. More research is needed to better understand and explain cultural differences where they occur.

It is very informative that hospitality is so consistently associated with all forms of wellbeing - social wellbeing, subjective wellbeing, and general flourishing. It may be that hospitality contributes to wellbeing, or that it follows from it, and it is possible that the influence is bi-directional. It is also informative that hospitality is seen in generally positive ways across diverse cultures, and yet is most strongly associated with specific personality characteristics. The direction of causal influence and cultural variation in hospitality are both fruitful areas for future research.

An important task for future research will be to explore whether hospitality raises wellbeing, or whether people high in wellbeing simply tend to be more hospitable, or both. Alternately, a person's social feelings in general could influence both wellbeing and hospitality. Because the associations with flourishing and wellbeing on the one hand, and hospitality on the other, were so robust, and found across very diverse cultures, this link is important to understand. We particularly hope that future researchers will investigate the conceptual boundaries of hospitality. It will be interesting and useful to better understand how hospitality relates to generosity, altruism, organizational citizenship, conflict resolution and other social concepts. Ultimately, our hope is that our results alert scholars to the importance of hospitality, as well as to a tool to study it.

\section{Authors}

Robert Biswas-Diener

Noba

robert@nobaproject.com

Kostadin Kushlev

Georgetown University

Rong Su

University of Iowa

Fallon Goodman

George Mason University

Todd Kashdan

George Mason University 


\section{Ed Diener}

University of Virginia, University of Utah and the Gallup Organization

\section{Publishing Timeline}

Received 16 January 2019

Accepted 2 May 2019

Published 9 July 2019

\section{References}

Batson, C. D., Early, S., \& Salvarani, G. (1997). Perspective taking: Imagining how another feels versus imaging how you would feel. Personality and Social Psychology Bulletin, 23(7), 751-758.

Baumgardner, S. R., \& Crothers, M. K. (2009). Positive psychology. Upper Saddle River, NJ: Prentice Hall/Pearson Education.

Diener, E., \& Seligman, M. E. P. (2002). Very happy people. Psychological Science, 13(1), 81-84. http://doi.org/10.1111/1467-9280.00415

Diener, E., Wirtz, D., Tov, W., Kim-Prieto, C., Choi, D., Oishi, S., \& Biswas-Diener, R. (2009). New measures of well-being: Flourishing and positive and negative feelings. Social Indicators Research, 39, 247-266.

Diener, E. D., Emmons, R. A., Larsen, R. J., \& Griffin, S. (1985). The Satisfaction with Life Scale. Journal of Personality Assessment, 49(1), 71-75.

Dienhart, J. R., Gregoire, M. B., Downey, R. G., \& Knight, P. K. (1992). Service orientation of restaurant employees. International Journal of Hospitality Management, 11 (4), 331-346.

Donnellan, M. B., Oswald, F. L., Baird, B. M., \& Lucas, R. E. (2006). The mini-IPIP scales: Tiny-yeteffective measures of the Big Five factors of personality. Psychological Assessment, 18(2), 192-203.

Epley, N., \& Schroeder, J. (2014). Mistakenly seeking solitude. Journal of Experimental Psychology: General, 143(5), 1980-1999. http://doi.org/10.1037/a0037323

Grobelna, A. (2015). Extraversion and its importance in the hospitality workplace. Analyzing the selected job outcomes. Economic Problems of Tourism, 3(31), 105-124.

Hodges, S., \& Biswas-Diener, R. (2007). Balancing the empathy expense account: Strategies for regulating empathic response. In T. F. D. Farrow \& P. W. R. Woodruff (Eds.), Empathy in mental illness and health (pp. 389-407). Cambridge, UK: Cambridge University Press.

Hogan, J., Hogan, R., \& Busch, C. M. (1984). How to measure service orientation. Journal of Applied Psychology, 69(1), 167-173.

InterNations (2018). Expat Insider 2018. www.internations.org/expat-insider

Jónasson, ++B. (1992). Hávamál: The sayings of the Vikings. Reykjavík, Iceland: Gudrun.

Kahneman, D., Krueger, A. B., Schkade, D. A., Schwarz, N., \& Stone, A. A. (2004). A survey method for characterizing daily life experience: The day reconstruction method. Science, 306(5702), 1776-1780. http://doi.org/10.1126/science.1103572

Pavot, W., \& Diener, E. (1993). Review of the Satisfaction with Life Scale. Psychological Assessment, 5(2), 164-172.

Peterson, C. (2006). A primer in positive psychology. New York, NY: Oxford University Press.

Peterson, C., \& Seligman, M. E. (2004). Character strengths and virtues: A handbook and classification. New York, NY: Oxford University Press.

Pezzotti, G. (2011). The essence of hospitality and service. In M. C. Sturman, J. B. Corgel \& R. Verma (Eds.), The Cornell school of hotel administration on hospitality: Cutting edge thinking and practice (pp. 518). Hoboken, NJ: John Wiley \& Sons. 
Reis, H. T., Sheldon, K. M., Gable, S. L., Roscoe, J., \& Ryan, R. M. (2000). Daily well-being: The role of autonomy, competence, and relatedness. Personality and Social Psychology Bulletin, 26(4), 419-435. http://doi.org/10.1177/0146167200266002

Rozin, P. (2006). Domain denigration and process preferences in academic psychology. Perspectives on Psychological Science, 1(4), 365-376.

Sandstrom, G. M., \& Dunn, E. W. (2014). Social interactions and well-being: The surprising power of weak ties. Personality and Social Psychology Bulletin, 40(7), 910-922. http://doi.org/10.1177/0146167214529799

Snyder, C. R., \& Lopez, S. J. (2002). Handbook of positive psychology. New York, NY: Oxford University Press.

Snyder, C. R., Lopez, S. J., \& Pedrotti, J. T. (2011). Positive psychology: The scientific and practical explorations of human strengths (2nd ed.). Thousand Oaks, CA: Sage.

Walker, J. R. (2009). Introduction to hospitality. London, England: Pearson.

\section{Appendix 1}

\section{The Brief Hospitality Scale}

Please answer openly and honestly to each question using the scale provided to indicate the amount you agree or disagree with each statement below:

7 - Strongly agree

6 - Agree

5 - Slightly agree

4 - Neither agree nor disagree

3 - Slightly disagree

2 - Disagree

1 - Strongly disagree

1. ___ I enjoy hosting others.

2. __ When I host others, I feel good about myself.

3. B__ Being hospitable is something that comes easily to me.

4. I I I am a very hospitable person.

* The authors of this scale grant permission for its use for both commercial and non-commercial purposes so long as attribution to the authors is made. 